\title{
Low-cost Implementations of NTRU for pervasive security ${ }^{*}$
}

\author{
Ali Can Atıc1 \\ Istanbul Technical University \\ Institute of Science and Technology \\ aticial@itu.edu.tr \\ Junfeng Fan \\ Katholike Universiteit Leuven \\ ESAT/COSIC and IBBT \\ junfeng.fan@esat.kuleuven.be
}

\author{
S. Berna Örs Yalçın \\ Istanbul Technical University \\ Faculty of Electrical and Electronics Engineering \\ siddika.ors@itu.edu.tr
}

\begin{abstract}
NTRU is a public-key cryptosystem based on the shortest vector problem in a lattice which is an alternative to RSA and ECC. This work presents a compact and low power NTRU design that is suitable for pervasive security applications such as RFIDs and sensor nodes. We have designed two architectures, one is only capable of encryption and the other one performs both encryption and decryption. The strategy for the designs includes clock gating of registers, operand isolation and precomputation. This work is also the first one to present a complete NTRU design with encryption/decryption circuitry. Our encryption-only NTRU design has a gate-count of 2.8 kgates and dynamic power consumption of $1.72 \mu W$. Moreover, encryption-decryption NTRU design consumes about $6 \mu W$ dynamic power and consists of 10.5 kgates.
\end{abstract}

\section{Introduction}

The feasibility of Public-key (PK) solutions for RFIDs and sensor networks is an open research problem due to severe limitations in costs, area and power. RFID tags and sensor nodes are extreme examples as they imply very

\footnotetext{
${ }^{*}$ This work is funded partially by IBBT, Katholieke Universiteit Leuven (OT/06/40) and FWO projects (G.0300.07 and G.0450.04). This work was supported in part by the IAP Programme P6/26 BCRYPT of the Belgian State (Belgian Science Policy), by the EU IST FP6 projects (ECRYPT) and by the IBBT-QoE project of the IBBT.
}

low budget for the number of gates, power, bandwidth etc. whilst they sometimes require security solutions. Implementations of Public-key Cryptography (PKC) are very difficult in those environments as PKC deploys computationally demanding operations. However, PKC protocols are useful for applications that need strong cryptography and services such as authentication, signatures, key-exchange etc. In addition, the use of PKC reduces power due to less protocol overhead [3].

In this paper, we present low-cost implementations of the Public-key algorithm NTRU, which are viable for RFIDs and sensor nodes. Section 2 investigates the previous implementations of low-cost PKC. Section 3 summarizes the basics of NTRU algorithm such as key generation, encryption and decryption. Section 4 gives brief information about the low-power design methods used in the designs. Section 5 gives the details of the architecture of encryption-only NTRU whereas, section 6 shows the architecture of encryption/decryption NTRU. Section 7 discusses the power, area and latency results of our implementation and previous implementations. And finally, Section 8 concludes the paper.

\section{Previous work}

To the best of our knowledge very few papers discuss the possibility for PKC (specifically NTRU) for RFIDs and sensor nodes. In the master's thesis of O'Rourke [7], a hardware core is designed which performs only NTRU polynomial multiplication. It has a gate count of minimum 1483 
gates, but the design is not optimized for low-cost and there is no power consumption data. Another NTRU design is implemented by Bailey et al. [1], and it performs only encryption. The design has approximately 60000 gates in a Xilinx Virtex 1000EFG860 FPGA. The most detailed low-cost implementation of NTRU is realized by Kaps [6]. The author investigated implementations of two algorithms: Rabin's scheme and NTRUEncrypt. It is concluded that NTRUEncrypt features a suitable low-power and small footprint solution with a total complexity of 3000 gates and power consumption of less than $20 \mu \mathrm{W}$ at $500 \mathrm{kHz}$. As one would expect, he showed that Rabin's scheme is not a feasible solution. Apart from NTRU implementations, there also exist a compact ECC implementation in [2]. In this work, the best solution has 6718 gates for modular arithmetic unit and control unit (data memory not included), and it consumes power less than $30 \mu W$ at $500 \mathrm{kHz}$.

\section{NTRU algorithm}

NTRU [4] is a public-key cryptosystem based on the shortest vector problem in a lattice. Basic operations of NTRU are realized in a truncated polynomial ring $R=$ $Z[X] /\left(X^{N}-1\right)$. Polynomials in the ring have integer coefficients and a degree of $N-1$. Addition is carried out in a normal way by adding the coefficients that have the same degree while multiplication is carried out in the following way. During multiplication the rule $X^{N} \equiv 1$ is applied to all elements which have a degree equal or greater than $N$. This multiplication is called star multiplication [1] and denoted with the $*$ symbol. Thus, the product of two polynomials $a$ and $b$

$$
\begin{gathered}
a(X)=a_{0}+a_{1} X+a_{2} X^{2}+\cdots+a_{N-1} X^{N-1} \\
b(X)=b_{0}+b_{1} X+b_{2} X^{2}+\cdots+b_{N-1} X^{N-1}
\end{gathered}
$$

can be calculated as,

$$
\begin{gathered}
c(X)=a(X) * b(X) \\
c_{k}=a_{0} b_{k}+a_{1} b_{k-1}+\cdots+a_{N-1} b_{k+1}=\sum_{i+j \equiv k \bmod N} a_{i} b_{j}
\end{gathered}
$$

\begin{tabular}{|c|c|c|c|c|c|}
\hline$\times$ & $\begin{array}{l}a_{4} \\
b_{4}\end{array}$ & $\begin{array}{l}a_{3} \\
b_{3} \\
\end{array}$ & $\begin{array}{l}a_{2} \\
b_{2} \\
\end{array}$ & $\begin{array}{l}a_{1} \\
b_{1} \\
\end{array}$ & $\begin{array}{l}a_{0} \\
b_{0}\end{array}$ \\
\hline \multirow{6}{*}{+} & $a_{4} b_{0}$ & $a_{3} b_{0}$ & $a_{2} b_{0}$ & $a_{1} b_{0}$ & $a_{0} b_{0}$ \\
\hline & $a_{3} b_{1}$ & $a_{2} b_{1}$ & $a_{1} b_{1}$ & $a_{0} b_{1}$ & $a_{4} b_{1}$ \\
\hline & $a_{2} b_{2}$ & $a_{1} b_{2}$ & $a_{0} b_{2}$ & $a_{4} b_{2}$ & $a_{3} b_{2}$ \\
\hline & $a_{1} b_{3}$ & $a_{0} b_{3}$ & $a_{4} b_{3}$ & $a_{3} b_{3}$ & $a_{2} b_{3}$ \\
\hline & $a_{0} b_{4}$ & $a_{4} b_{4}$ & $a_{3} b_{4}$ & $a_{2} b_{4}$ & $a_{1} b_{4}$ \\
\hline & $c_{4}$ & $c_{3}$ & $c_{2}$ & $c_{1}$ & $c_{0}$ \\
\hline
\end{tabular}

In other notation, if we write the polynomials $a, b$ and $c$ as coefficient vectors, then, the result $c=a * b$ simply equals to convolution product of two vectors as shown below [7]:

NTRU public-key cryptosystem has three integer parameters $(N, q, p)$ and four sets $L_{f}, L_{g}, L_{r}, L_{m}$ of polynomials of degree $N-1$ which have all integer coefficients. It is assumed that $N$ is prime, $\operatorname{gcd}(p, q)=1$ and $q$ is always fairly larger than $p$. NTRU with $N=263$ provides an equivalent security level of 1024-bit RSA and 160 bits long ECC $[5,10]$.

Key Generation: To generate key sets of NTRU, one must first choose two random polynomials $f \in L_{f}$ and $g \in L_{g}$. These two polynomials must be small polynomials which means their coefficients must be much smaller than $q$. Besides, $f_{q} \equiv f^{-1}(\bmod \mathrm{q})$ and $f_{p} \equiv f^{-1}(\bmod \mathrm{p})$ must exist. Then, the following computation is performed, $h \equiv f_{q} * g(\bmod \mathrm{q})$. Now $h$ is our public key while $f$ and $f_{p}$ are our secret keys. For, more information about parameter selection, small polynomials and finding inverses of polynomials we refer to [4, 8, 9].

Encryption: Firstly, a message $m$ which is a small polynomial is chosen from the plaintext set $L_{m}$ and then a small random polynomial $r$ is chosen from the set $L_{r}$ as blinding value. Finally, encrypted text is evaluated as, $e \equiv p r * h+m(\bmod \mathrm{q})$. During encryption, $h$ will be always multiplied by $p$. So, to avoid unnecessary computation it is suggested to use $h \equiv p f_{q} * g(\bmod q)[11]$.

Decryption: In order to decrypt the encrypted text $e$, one must first compute $a \equiv f * e \equiv p r * g+f * m(\bmod \mathrm{q})$. At this stage it is essential to chose the coefficients of $a$ between $-q / 2$ and $q / 2$ instead of 0 and $q-1$. Otherwise, message may not be properly recovered. After this step, $b \equiv a(\bmod \mathrm{p})=f * m$ should be calculated. As the final step, message can be recovered by the multiplication of $f_{p}$ and $b$ modulo $p, c \equiv b * f_{p}(\bmod \mathrm{p})=m$.

\section{Low power design methods}

There are many power reduction methods available for both technological and architectural level. Here, we will mention only the techniques we have used.

The circuit is planned to be running at low frequencies $(500 \mathrm{kHz})$ thus, we have used a low leakage technology library for synthesizing our design, which enabled us to obtain considerably lower static power consumptions. At the same time, we have also used certain methods to decrease the dynamic power consumption such as clock gating, operand isolation and precomputation.

The aim of clock gating is to control the clock input of the registers by an enable signal and isolating the clock input unless new values are loaded to registers and decreasing the switching activity by this way. In clock gating circuit [13], it is preferred to use a latch in order to disable glitches which may occur in the clock inputs of registers. Figure 1 shows a clock gated register.

Operand isolation is another way of reducing the switching activity by isolating the input of complex combinational 


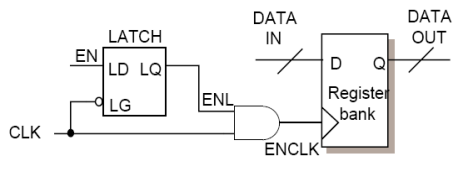

Figure 1. A gated register

circuits (i.e. multipliers, adders) when their outputs are not used.

To avoid consuming power for key generation, public key $h \equiv r * p f_{q} * g(\bmod \mathrm{q})$ is precomputed and stored in the circuit, since we assume that $h$ remains same at each encryption.

\section{Implementation of encryption-only NTRU}

We have chosen $N=167, q=128$ and $p=3$ among the parameter sets of NTRU that yields to a high level of security [4]. As a result of this choice public key $h$ has coefficients in the interval $[0,127]$, random polynomial $r$ and message $m$ have coefficients from the set $\{-1,0,1\}$. Thus coefficients of $h$ are encoded in 7 bits, and coefficients of $r$ and $m$ are encoded in 2 bits. And, while dealing with negative numbers, two's complement representation is used.

The main architecture of the encryption engine, as shown in Figure 2, consists of a look-up table to store $h$, a polynomial multiplier (PM) to perform the star multiplication, a Partially Rotating Register (PRR) $(N \times 2$ bits wide) to hold and rotate $r$ and a control logic that manages the whole encryption process.

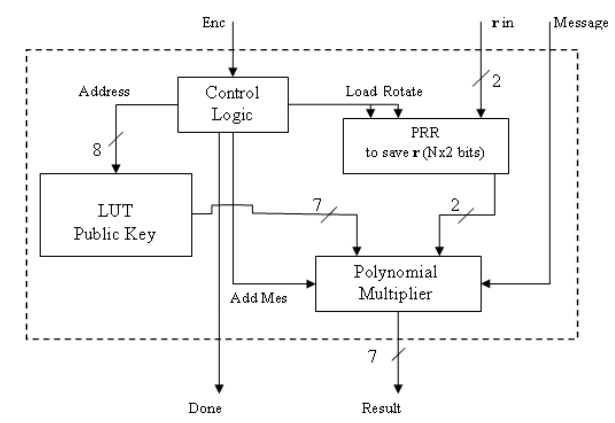

Figure 2. NTRU encryption architecture

In this design we do not consider generation of random polynomials, so we assume that we are able to receive random polynomial coefficients one by one from the input $r_{i n}$.

\subsection{Look-up table}

The Look-up table (LUT) is implemented in a completely combinational way, in order to give out the appropriate public key coefficients according to its address input.

\subsection{Polynomial multiplier}

This is the main arithmetic core where all computations are carried out. It has a 7-bit multiplier, a 7-bit Carry Lookahead Adder (CLA), and a 7-bit register as shown in Figure 3.

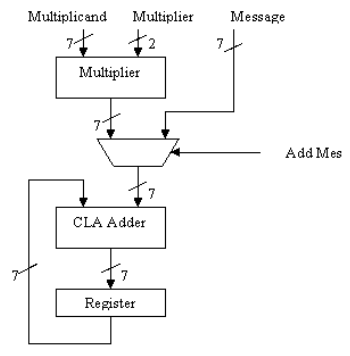

Figure 3. Polynomial multiplier unit

All the submodules of PM are realized for 7 bit computations since we need to reduce the results modulo 128 . This way, intermediate and final results are directly reduced during computations.

Multiplier has such a simple structure owing to small coefficients of random polynomial $r$. Since the coefficients of $r$ take values from the set $\{-1,0,1\}$, the product for a multiplicand $x$ is easily computed from $\{-x, 0, x\}$. In case of negative multiplicand, the product is generated by inverting the bits and making the carry input of the CLA adder 1.

CLA adder is chosen because of its non-carry propagation property which we need for less switching activity. At the same time, not to increase the area, the CLA is implemented in two blocks. Intermediate products are saved in the register, and partial sum value is always fed back to one input of CLA adder. After all multiplication and accumulation steps, message input is connected to one input of the adder and final result is generated.

\subsection{Partially rotating register}

Partially rotating register is a modified version of regular rotating register. We made a modification to benefit from clock gating maximally. During encryption, random polynomial coefficient at the input of PM is changing for every new public key value. So in case of a regular register, whole bits must be shifted one time, which means switching activity of $N \times 2$ registers for every partial product computation. We have seen by our measurements that clock gating a regular rotate register does not have a positive effect on power consumption during NTRU encryption. On the contrary, it increases the dynamic power consumption due to extra switching activity comes from gating circuits (see Table 1). To overcome that negative situation we designed a partially rotating register, as illustrated in Figure 4. 


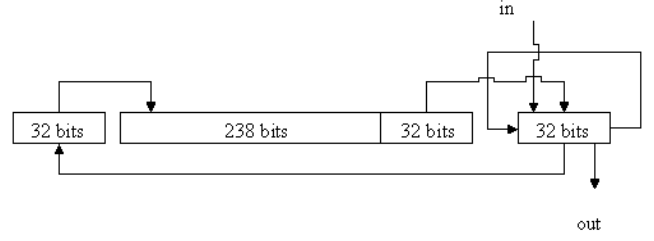

Figure 4. Partially rotating register

In this architecture the right hand side 32-bit register is the part that is always rotating during encryption and loading of random polynomial. During the loading stage most significant two bits of that register are used as input and the output is always the least significant two bits of that register. A partial rotate signal is sent by the controller till all 16 values of the register have been used and then a whole rotate signal is sent to renew the values of partial rotate register. After receiving that signal, circuit makes a block rotation with width of 32 bits. With this method, only 32 bits are switching constantly during encryption while the rest of the registers switch only 9 times, in the computation of each cipher text word.

\subsection{Control logic}

Controller of the encryption engine is designed with a finite state machine (FSM) which has four states. It controls the whole process by two 8-bit counters and one 4-bit counter. It starts with the initial state after reset and always checks the Enc input. If it detects a high signal it transits to the loading state. During loading state, the coefficients of $r$ are loaded to the PRR one by one. After loading all coefficients, FSM passes to the multiplication state.

For multiplication, the coefficients of $h$ and $r$ are multiplied and accumulated. Multiplication is followed by the add message state where message is added to the current sum, cipher text is given out and a done signal is made 1 for one clock cycle. After addition of the message it again transits to multiplication state to compute the next coefficient unless it is the last coefficient. After calculating last coefficient it transits to the initial state.

\section{Implementation of encryption-decryption NTRU}

The same parameter sets are used also for encryptiondecryption NTRU design. In this case, we have two more polynomials that are, private keys $f$ and $f_{p}$. $f$ has coefficients from the set $\{-1,0,1\}$ while $f_{p}$ has coefficients from the set $\{0,1,2\}$.

Figure 5 shows the architecture of the design. For simplicity not all of the modules and inner signals are shown. For decryption, a Mod-3 unit, two more look-up tables to store private keys $f$ and $f_{p}$, a $N \times 7$ bit PRR and a result register is added to the previous design.

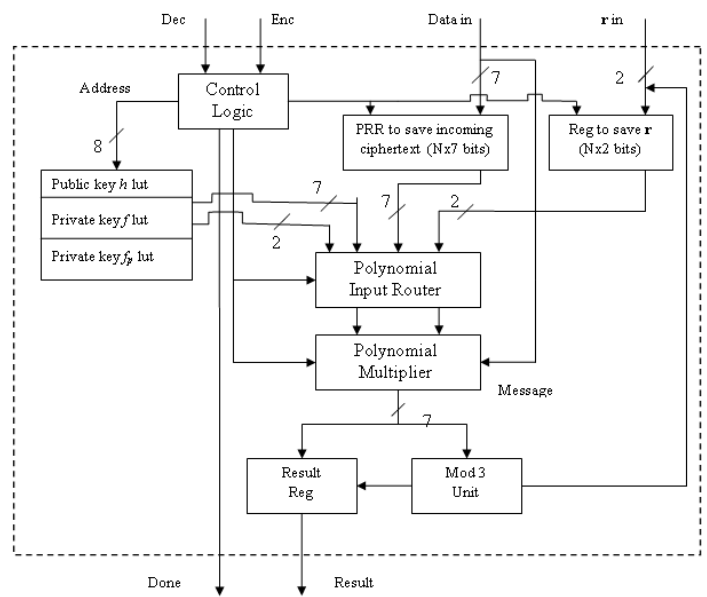

Figure 5. NTRU encryption decryption architecture

Moreover there are four routers, composed by multiplexers, which direct the correct values to the correct inputs according to encryption and decryption stage. During encryption, 7-bit and 2-bit input of PM are bounded to LUT $h$ and $N \times 2$ wide register outputs, respectively. Output of PM is connected to the result register. In the first multiplication of decryption, 7-bit and 2-bit input of PM are bounded to $N \times 7$ wide PRR and LUT $f$, respectively. And output of PM is connected to Mod-3 unit, while the output of Mod3 unit is connected to the input of register $N \times 2$. During second multiplication of decryption, 7-bit and 2-bit input of PM are connected to LUT $f_{p}$ and register $N \times 2$, respectively. Output of PM is again connected to Mod-3 unit but the output of Mod- 3 unit is bounded to result register.

The components used in the NTRU encryption engine are also used in this design. Again, we used look-up tables to store the public key and private keys. $N \times 7$ bits wide PRR has the same architecture as defined in Sect. 5, only with modified bit lengths. $N \times 2$ bits wide register is implemented as a regular left rotating register. Result register is also a regular register with a load signal.

The only change has occurred in PM. Since, we designed PM only working in modulo 128 , we added an over flow output, which is simply carry output of the CLA, to count the number of modulo 128 reductions during second multiplication of decryption which the computations should be done modulo 3. Also, in this design PM is capable of multiplying with 2 which is used during decryption process.

\subsection{Routers}

There are four routers in the design. One for address input of the look-up tables, one for input of the PM, one 
for rotating register inputs, and one for result register input. These modules maintain the necessary connections between modules according to the control signals generated by controller.

\subsection{Mod-3 Unit}

Reduction mod 3 is done by a finite state machine based circuit [12]. The FSM starts at state 0 and checks the bits from left to right and makes state transitions according to the value of that bit. The final state after checking the last bit is the value of the number in modulo 3 .

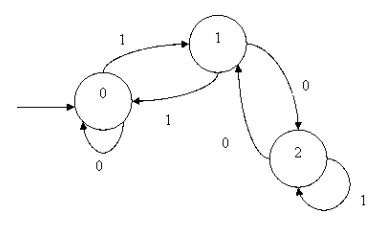

Figure 6. Mod-3 FSM

Overflow output of PM is also connected to this module. There is a 2-bit overflow counter that counts the occurrences of overflow. By using the value of this counter, we obtain the correct reduction mod 3 results.

\subsection{Control logic}

Controller of the design is implemented as an FSM that has seven states. It controls the state transitions with two 8bit counters and one 4-bit counter. The FSM starts with the initial state following up the reset and directly transits to the checking state by controlling the Enc and Dec inputs. Enc and Dec inputs are checked continuously at checking state. On detection of a high signal at one of these inputs, FSM transits to loading state, otherwise state remains unchanged.

During loading state, if it is encryption, coefficients of $r$ are loaded to the register which is $N \times 2$ wide, one by one. If it is decryption, coefficients of cipher text $e$ are loaded to the $N \times 7$ wide PRR, one by one. Loading is followed by the multiplication state. Multiplication state is common for encryption and decryption, since polynomial multiplication is carried out in the same way for both situations. After finishing the multiplication and accumulation of one coefficient it transits to add message state during encryption otherwise transits to reduction 3 state.

During add message state, message is added to the current sum and controller goes to final result state. At reduction 3 state the result generated by PM is loaded in Mod-3 unit and the modulo 3 value of that number is calculated. Both for the first and second reduction $\bmod 3$, controller goes to final result state after computation.

At final result state, if the circuit is doing encryption, controller loads the result to result register and makes the done output 1 for one clock cycle. If decryption is being carried out and circuit is performing the first multiplication, FSM loads the reduction 3 result to $N \times 2$ wide register. If the second multiplication is being carried out, then reduction 3 result is loaded into result register and done output is made 1 for one clock cycle. For all situations if the last coefficient is given out, system transits to initial state, otherwise, it transits to multiplication state.

\section{Analysis}

We wrote our designs in GEZEL [14] and optimized them for low power and low area. As the technology library, the Faraday Low Leakage $0.13 \mu \mathrm{m}$ library is used. Designs are synthesized by Synopsys Design Vision at $500 \mathrm{kHz}$ and average power measurements are done by Synopsys Power Compiler. All power measurements are done by using the switching activity which is captured by gate-level simulation with ModelSim. Since, the most detailed low-cost NTRU implementation belongs to the work of Kaps, we compare our results with his work [6].

We synthesized three different designs for encryption. One is without any enhancements for power consumption, one is with clock gated registers, and the last one is with clock gated registers and partially rotating registers. We will denote them as Enc1, Enc2 and Enc3 respectively. Gate counts of the designs found by dividing the whole area of the circuit by the area of one 2-input NAND gate.

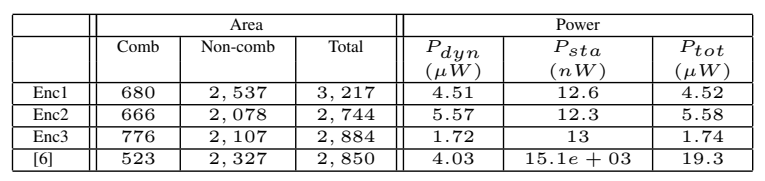

\section{Table 1. Power and area results of only en- cryption NTRU}

As we can see from Table 1, our most optimized design Enc3 and the previous work have almost same gate numbers whereas our design is superior to [6], with $1.72 \mu \mathrm{W}$ dynamic and $1.74 \mu W$ total power consumption. Owing to technology library that has $p W$ as the leakage power unit, we also measured considerably lower static power consumptions. Since the rotating register is the major source of area $(73.6 \%)$ and power $(82.6 \%)$ consumption in Encl, using partially rotating registers enables us to obtain dynamic power savings of more than $50 \%$. Furthermore, total power consumption of the Enc3 design during idle state is measured as $0.18 \mu W$.

Our design finishes encryption at $N \times(N+1)+N$ clock cycles. For our case $N=167$ and with a clock frequency of $500 \mathrm{kHz}$, it takes $56.44 \mathrm{~ms}$ (28 223 clock cycles), which is $3.5 \%$ faster than [6] that finishes encryption at $58.45 \mathrm{~ms}$ (29225 clock cycles). 
To the best of our knowledge, no encryption-decryption NTRU design is reported before, so we only give our results. Table 2 shows the consumption of optimized design. As seen from the table total gate count is 10.5 kgates and $84 \%$ of the area is occupied by registers.

\begin{tabular}{|l||c|c|c|c|}
\hline Block & Comb & Non-comb & Total & $\%$ \\
\hline Controller & 231 & 172 & 403 & 3.8 \\
\hline Luts & 717 & 0 & 717 & 6.8 \\
\hline PM & 109 & 81 & 190 & 1.8 \\
\hline Reduction 3 & 66 & 102 & 168 & 1.2 \\
\hline$N \times 2$ & 33 & 1877 & 1910 & 18.2 \\
\hline$N \times 7$ & 388 & 6473 & 6961 & 66.3 \\
\hline Others & 104 & 42 & 146 & 1.4 \\
\hline Total & 1651 & 8848 & 10500 & 100 \\
\hline
\end{tabular}

\section{Table 2. Area consumption of encryption- decryption NTRU}

Power consumption of the circuit is measured for three different working states of the design: Encryption, decryption and idle. Table 3 shows these results.

As seen our optimized design consumes only about $6 \mu W$ while encryption and decryption and $0.5 \mu W$ during idle state. Rotating registers are the major sources that consume power. Around $80 \%$ of the power is consumed by these registers during encryption and decryption.

For the encryption-decryption NTRU design, encryption takes $N \times(N+2)+N$ clock cycles while decryption takes $2 \times N \times(N+11)+N$ clock cycles. For the case $N=167$ and with a clock frequency of $500 \mathrm{kHz}$, encryption takes $56.78 \mathrm{~ms}$ ( 28390 clock cycles), decryption takes $119.23 \mathrm{~ms}$ (59 619 clock cycles).

\section{Conclusion}

In this paper we presented a compact and low power NTRU design that is suitable for pervasive security applications such as RFIDs and sensor nodes. We designed two architectures. One is only capable of encryption and the other one performs both, encryption and decryption. Our encryption-only NTRU design has a gate-count of 2.8 kgates, which makes it a very compact solution. The encryption takes $56.44 \mathrm{~ms}$ and this makes it $3.5 \%$ faster than the best previous work. Our design consumes $1.72 \mu \mathrm{W}$ of dynamic power. This presents a saving of a factor more than 2 , when compared with the previous work. This work is also the first one to present a complete NTRU design with encryption/decryption circuitry.We obtained a gate count of 12.3 kgates for the design, which we further improved to 10.5 kgates. The optimized design has the following results, $5.93 \mu W, 6.04 \mu W$ and $0.45 \mu W$ for dynamic power consumption during encryption, decryption and idle state, respectively. The latency for encryption and decryption, takes $56.78 \mathrm{~ms}$ and $119.23 \mathrm{~ms}$ respectively.

\begin{tabular}{|c|c||c|c|c|}
\hline & \multicolumn{1}{|c||}{} & \multicolumn{3}{c|}{ Power } \\
\cline { 2 - 5 } & $P_{d y n}(\mu W)$ & $P_{s t a}(n W)$ & $P_{\text {tot }}(\mu W)$ \\
\hline \multirow{3}{*}{ NTRU Plain } & Encryption & 12.3 & 49.4 & 12.4 \\
\cline { 2 - 5 } & Decryption & 15.9 & 50.5 & 16 \\
\cline { 2 - 5 } & Idle & 10.1 & 49.7 & 10.2 \\
\hline \multirow{3}{*}{ NTRU Opt. } & Encryption & 5.93 & 46.6 & 5.98 \\
\cline { 2 - 5 } & Decryption & 6.04 & 50.8 & 6.11 \\
\cline { 2 - 5 } & Idle & 0.45 & 46.3 & 0.5 \\
\hline
\end{tabular}

Table 3. Power results of encryption decryption NTRU

Furthermore, in order to speed up the designs more PM units may be used in parallel with a little change in the rest of the design. Also, it is possible to reduce the multiplication number needed for decryption from 2 to 1 , by selecting algorithm parameters in a different way.

\section{References}

[1] D. Bailey, D. Coffin, A. Elbirt, J. Silverman, and A. Woodbury. NTRU in Constrained Devices. In Cryptographic Hardware and Embedded Systems, Paris, France, 2001.

[2] L. Batina, N. Mentens, K. Sakiyama, B. Preneel, and I. Verbauwhede. Low-cost elliptic curve cryptography for wireless sensor networks. In 4th European Workshop on Security and Privacy in Ad hoc and Sensor Networks, Lecture Notes in Computer Science, volume 4537, pages 6-17. SpringerVerlag, 2006.

[3] G. Gaubatz, J.-P. Kaps, E. Öztürk, and B. Sunar. State of the art in ultra-low power public key cryptography for wireless sensor networks. In Third IEEE Int. Conf. Pervasive Comput. Commun. Workshops, volume v2005, pages 146-150. IEEE Computer Society, Mar 2005.

[4] J. Hoffstein, J. Pipher, and J. H. Silverman. NTRU: A RingBase Public Key Cryptosystem. In J. P. Buhler, editor, Algorithmic Number Theory (ANTS III), Lecture Notes in Computer Science, volume 1423, pages 267-288, Berlin, 1998. Springer-Verlag.

[5] N. Howgrave-Graham, J. H. Silverman, and W. Whyte. Choosing Parameter Sets for NTRUEncrypt with NAEP and SVES3. In Topics in Cryptology CT-RSA 2005, Lecture Notes in Computer Science, volume 3376, pages 118-135, Berlin, 2005. Springer.

[6] J. Kaps. Cryptography for Ultra-Low Power Devices. PhD thesis, Worcester Polytechnic Institute, May 2006.

[7] C. M. O'Rourke. Efficient NTRU Implementations. Master's thesis, Worcester Polytechnic Institute, April 2002.

[8] J. H. Silverman. Invertibility in Truncated Polynomial Rings. Technical report, NTRU Cryptosystems, 1998.

[9] J. H. Silverman. Almost Inverses and Fast NTRU Key Creation. Technical report, NTRU Cryptosystems, 1999.

[10] http://www.ntru.com/cryptolab/faqs.htm\#sixteen.

[11] The NTRU Public Key Cryptosystem A-Tutorial.

[12] www.zenoli.net/category/mathematics/, 2007.

[13] Synopsys, Inc. Power Compiler User Guide, 2006.

[14] http://rijndael.ece.vt.edu/gezel2/index.php. 